\section{The Gut Microbiome and Immune Modulation in The Treatment of Malignancy}

\section{Abstract}

The potential role of the gut microbiomes in the response and toxicity to immune checkpoint inhibitor therapies (ICls) in advanced malignancies has been a growing area of interest within the field of medical oncology. A number of pre-clinical and clinical trials have identified different microbiome factors that beneficially impact upon $\mathrm{ICl}$ outcomes, including specific microorganisms and diversity and suggested that treatment outcomes can be influenced by modification of the gut microbiome, such as through antibiotic administration. There is coexisting evidence the microbiome may also impact on the toxicity profile of ICls. Currently, the available literature describes associations between the microbiome and $\mathrm{ICI}$ outcomes, but the causal link is yet to be established. Additionally, the studies to date pose problems in the inherent heterogeneity that exists between subjects and respective microbiome composition. While promising, murine-humanised models or germ-free mice do not necessarily exhibit comparable immunocompetency or metagenomic function to humans. The faecal microbiome is likely to play a part of the much larger anti-tumour immune response and patient factors that influence this, which must be viewed holistically in the clinical context. Ultimately, this is a promising area, hurtling forward rapidly. Research is equally underway for optimizing methods to administer treatments to alter these microbiomes, whether it be via faecal transplantation, or supplementation with short chain fatty acids directly to the bowel. Learning more about how the constituent parts of the microbiome exert local and systemic immune responses could herald a significant leap forward in how solid tumours are treated with immunotherapy.

Keywords: Immunotherapy; Microbiome; Immune checkpoint; Melanoma
Babu HS ${ }^{1}$, West ${ }^{2}$, Cripps A $^{2}$, Sanmugarajah $\mathbf{J}^{1,2}$, Mason $\mathbf{R}^{1}$

1 Department of Medical Oncology, Gold Coast University Hospital, Southport, QLD 4215, Australia

2 Griffith University, Gold Coast Campus, Parklands QLD 4222, Australia

*Corresponding author:

Dr. Robert Mason

\section{Robert.Mason@health.qld.gov.au}

Department of Medical Oncology, Gold Coast University Hospital, Southport, QLD 4215, Australia.

Tel: +61 1300744284

Citation: Babu HS, West N, Cripps A, Sanmugarajah J, Mason R (2019) The Gut Microbiome and Immune Modulation in The Treatment of Malignancy. Insights Biomed Vol.4 No.2:11

\section{Introduction}

Immune checkpoint inhibitors (ICls) including anti-PD1/PDL1 and anti-CTLA monoclonal antibodies have revolutionized the treatment of several advanced malignancies including melanoma, lung, kidney, bladder and head and neck and skin cancer [1-8]. They are associated with a significant and durable improvement in survival in these cancer types and have a novel spectrum of immune-related toxicities. However, many patients will not respond to treatment and further treatment options often have limited sustained efficacy. For patients with advanced cancers failing to respond to ICls, there is an urgent clinical need for treatment options and the ability to restore a durable antitumor immune response.

Part of the burgeoning body of research underway considers new methods to modulate the anti-tumor immune response in conjunction or following aforementioned therapies. One particular field of interest involves the interaction between the gut microbiome, tumor immune profile and characteristics, and the host (patient) immune phenotype and response. The gut plays host to a diverse number of organisms, including bacteria, viruses, archaea, protozoa, yeast and fungi; a significant proportion of research has been devoted to studying the relationship between the microbiome and $\mathrm{ICl}$ treatment outcomes and interventional studies are now in development.

\section{Literature Review}

\section{The gut microbiome}

The gut microbiome and the human body demonstrate a mutualistic relationship. The microbiome contributes towards the development and integrity of the immune system and helps the 
host to acquire nutrients that it would otherwise not be able to access $[9,10]$. As such, the make-up and subsequent manipulation of intestinal flora for health outcomes has been a point of interest [11]. Modalities that have been investigated include the following: prebiotics, food compounds used selectively to grow or influence specific microorganisms in the gut; probiotics, which are live microorganisms intended to provide health benefits in sufficient quantities via specific improvements to gut flora; or synbiotic, a combination of the two [12-14]. Several different interactions have been considered between the microbiome and malignancy. Specific bacteria have been implicated in cancer initiation, development and progression, but additionally, a role has been suggested in the efficacy and toxicity of $\mathrm{ICl}$ by the microbiome $[15,16]$.

High circulating levels of SCFAs (short-chain fatty acids), which are bacterial metabolites produced by microbiota fermentation of dietary fiber, have also been associated with enhanced generation of macrophage and dendritic cell precursors in the bone marrow [17]. SCFAs have also been shown to provide the capability of modulating populations of favorable bacteria in the gut, and it has been demonstrated that large quantities of SCFAs can be delivered to the large bowel via consumption of starch supplements (such as actylated and butyrylated starches) $[12,18]$. High levels of red meat consumption are known to be associated with an increased risk of colorectal cancer, and resistant starch consumption has been shown to confer some level of protection; mechanisms of actions include changes to the microbiota in the presence of the increased SCFAs produced from the starch substrate, and reduced levels of micro RNA expression via the SCFAs produced $[19,20]$. Increased intestinal permeability through upregulation and increased phosphorylation of key tight junction proteins, regulation and expansion of FoxP3+ regulatory T-cells and epigenetic modification of epithelial and immune cells have also been reported with the administration of butyrylated starches [21,22].

\section{Immune checkpoint inhibition and the microbiome}

The advent of $\mathrm{ICl}$ has heralded a new era of possibility in the treatment of cancer. Durable remissions in many different malignancies, including RCC, NSCLC and metastatic melanoma have been demonstrated. This is, however, not a consistent phenomenon, and some patients do not show response to $\mathrm{ICl}$. Innate mechanisms have been considered, including immune checkpoint independent immune suppression, low tumour antigen load, poor antigen expression and low mutational burdens, amongst others [23]. The interaction between the host immune system and the faecal microbiome has been identified, in preclinical and early clinical models, to relate to response to immunotherapy. There is some evidence also in inflammatory bowel disease (a disease with many striking similarities to the immune related colitis seen with PD-1 and CTLA4 inhibitors), that the faecal microbiome plays a significant role in the development and regulation of the disease. The use of the microbiome as a potential biomarker for the development of an immune related colitis is yet to be explored in a clinical context [24].

Pre-clinical studies have investigated this relationship; a study by Sivan et al. observed subcutaneous melanoma growth in mice
[25]. 16S ribosomal RNA sequencing identified Bifidobacterium as being positively correlated with improved tumour control. Oral administration of the probiotic demonstrated tumour control comparable to administering PD-L1 specific ICl, suggesting the possibility of spontaneous immune responses to tumour cells. The combination of the two resulted in near complete eradication of the tumour outgrowth. The results were attributed to a number of modulation factors, including upregulation of gene transcripts involved in CD8+ T-cell activation, co-stimulation and improved effector function, increases in major histocompatibility complex (MHC) Class II dendritic cells, increases in interferon y (IFN-y) producing tumour-antigen-specific T-cells, dendritic cell maturation, antigen processing and cross presentation, and chemokine-mediated recruitment of immune cells to the tumour microenvironment. An additional interesting observation from this study was that the mice were grown at different animal facilities but had different tumour growth rates despite being genetically identical and having identically implanted tumours. The differences in tumour growth rates were ablated following cohousing or faecal transfer [25].

Another study by Matson et al. examined germ-free mice with melanoma and change in treatment efficacy after administration of faecal transplantations from patients with advanced malignancy treated with PD-1 inhibitors including responding and non-responding patients [26]. Some bacteria, including Bifidobacterium longum, Collinsella aerofaciens and Enterococcus faecium were among the more prominent constituents of the responders' microbiomes. The mice receiving faecal transplants from responders were noted to have better outcomes comparably. In this study, SIY-specific CD8+ T-cells were shown in greater numbers in the responder group, but not FoxP3+CD4+ regulatory T-cells. This is consistent with increased priming of tumour antigen-specific CD8+ T-cells [26].

Vetizou et al. examined tumours in antibiotic-treated or germfree mice. CTLA-4 ICl response was poor due to depleted numbers of effector T-cells and tumour-infiltrating lymphocytes, but response was restored following oral administration of Bacteroides spp. or Burkholderia spp [27]. The study found that the microbiota composition affected interleukin-12 (IL-12) dependent $\mathrm{TH} 1 \mathrm{immune}$ responses [27]. These studies add to the existing literature with regards to how the microbiome impacts upon systemic immunity. Circulating bacteria-derived molecules are thought to drive immune stimulation and recognition of malignancy through molecular mimicry, as innate immune cells express pattern recognition receptors, which directly sense these products and subsequently modulate myelopoiesis and granulopoiesis via MyD88 dependent pathways [28].

These preclinical models have since paved the way for clinical studies. Routy et al. observed patients with non-small cell lung cancer and renal cell carcinoma and found a direct positive correlation between clinical responses to $\mathrm{ICl}$ and Akkermansia muciniphilia [24]. Gopalakrishnan et al. conducted a prospective study of a cohort of 112 melanoma patients undergoing PD-1 ICl, analysing the oral and gutmicrobiomes of patients [29]. Responders to $\mathrm{ICl}$ demonstrated a significantly higher alpha diversity in the gut microbiome compared to non-responders, and a prolonged of progression-free survival. Interestingly, the oral microbiome 
appeared to be distinct from that of the gut, harbouring a higher abundance of Lactobacillales (versus Bacteroidales in the faecal microbiome), and no significant differences in the oral microbiome were noted between responders and non-responders. Findings suggested that a higher diversity and abundance of Ruminococcacaea and Faecalibacterium, helping to constitute a "favourable" microbiome, were associated with a better response to immunotherapy, mediated by increased antigen presentation and a better $T$ cell effector function in both the periphery and the tumour microenvironment. Non-responders, on the other hand, were found to have a lower diversity of their microbiome and a greater number of Bacteroidales. This resulted in worse systemic and anti-tumour responses, attributable to poorer intra-tumoural lymphoid and myeloid proliferation, and a comparatively limited capacity for antigen presentation [29].

\section{Antibiotics and immune checkpoint inhibitor outcomes}

The role of antibiotics is an important consideration. Antibiotics are known to have a significant impact upon both developing and mature microbiomes, with lasting effects of dysbiosis [30]. Routy et al. observed the disruption of the microbiome by antibiotics and subsequent response to immunotherapy [24]. In this study, initially mice reared in specific pathogen-free conditions were administered 14 days of broad-spectrum antibiotics (ampicillin, colistin and streptomycin) concomitantly with ICls. Both survival and anti-tumour effects were reduced in these mice, compared to those that only received ICls. In patients, progression-free and overall survival were considerably shorter in those who had received antibiotics (beta-lactam inhibitors, fluoroquinolones or macrolides) either 2 months before or 1 month after initiation of $\mathrm{ICl}$ therapy. These were generally prescribed for common indications, such as dental, urinary and pulmonary infections. Univariate and multivariate Cox regression analyses demonstrated antibiotic use to be a predictor of resistance to PD-1 inhibitors in this population, independent of typical prognostic markers and this unfavourable effect was further confirmed with a validation cohort of 239 advanced non-small cell lung cancer patients receiving PD-1/PDL1 inhibitors for advanced cancer [24]. Another study by Derosa et al. corroborated these findings and observed that patients with both renal cell carcinoma and non-small cell lung cancer had worse progression free and overall survival outcomes if they took antibiotics within 30 days of treatment initiation. Multivariate analyses showed that the impact of antibiotics was significant for progression free survival in renal cell carcinoma, and for overall survival in non-small cell lung cancer [31].

\section{Immune checkpoint inhibitor toxicity and the microbiome}

Another important consideration includes toxicity associated with microbiomes and potential modulation. Immune-related colitis (ir-colitis) is a well described ICl-related adverse event (irAE), and the effect of gut microbiomes on toxicity have been investigated in both animals and humans [32]. There is evidence from inflammatory bowel disease (IBD) and irritable bowel syndrome (IBS) literature to support the notion that the specific organisms within the composition of the microbiome have a role in instigating ir-colitis [33-35]. In conjunction with existing studies that have specifically observed ir-colitis, this literature suggests that the gut microbiome composition may be a predictor of toxicity and clinical response to ICls. Though the contexts of IBD and ir-colitis are separate, common organisms were identified as being associated with both beneficial and undesirable outcomes, particularly Firmicutes and Bacteroidetes respectively. One study demonstrated the phylum Firmicutes in greater numbers within the microbiome of healthy subjects versus those that had irritable bowel syndrome, and another demonstrated an associated between enterotoxigenic Bacteroidetes and active IBD $[34,35]$. Considering Bacteroidetes, a study of melanoma patients on CTLA-4 inhibitors with Bacteroidetes-abundant microbiomes appeared to have reduced rates of ir-colitis. However, the authors outlined the challenges associating ir-colitis to specific organisms within the microbiome because the use of immune-modulating agents for the management of ir-colitis may also influence the faecal microbiome [36].

Another study demonstrated that patients with a higher abundance of Faecali bacterium and other Firmicutes and lower abundance of Bacteroides following CTLA-4 inhibitors were at greater risk of developing colitis. The same group were noted to have a higher rate of clinical response to $\mathrm{ICl}$, exemplified by longer progression-free survival and overall survival. The microbiome composition remained unchanged with administration of CTLA4 inhibitor therapy however, ir-colitis was associated with a decrease in bacterial diversity, particularly with respect to the Firmicutes phylum. Importantly, the sub-population of patients with a higher faecal Bacteroides proportion remained colitis-free but had poorer treatment outcomes [37]. Faecal transplantation is currently under investigation both to improve responses to $\mathrm{ICl}$ and for the treatment of immune-mediated colitis [38]. Though a biological link is not offered, these findings tie in with existing evidence that associates irAEs with treatment response [39].

\section{Future Perspectives}

The body of work to date does have limiting factors that must be acknowledged for the future. First and foremost, current evidence as described associates the gut microbiome constitution and level of biodiversity with $\mathrm{ICl}$ treatment response in advanced malignancy, however it is still not appreciated precisely how the tumour, gut microbiome and host immune response interact with one another to drive the development or regression of malignancy and other disease states. Furthering our knowledge of the underlying biological mechanisms that influence immune responses and treatment outcomes via the microbiome will inform the next phase of investigation, interventional studies.

Modalities to effectively deliver interventions have been described, including short chain fatty acids and faecal transplants, and other potential interventions, such as avoiding antibiotics, may be extrapolated from previous research. Given the number of variables involved, administering interventions to modulate the immune response ethically becomes difficult without appropriate prospective evaluations, to avoid harming patients by inducing an unfavourable response. Appropriate patient selection for interventional studies is therefore paramount.

Exploration of the role of the fecal microbiome is but one facet of research into the resistance of various malignancies to ICls and the 
negative regulation of these checkpoints remain but one element of the greater anti-tumour capability of the immune system. The mechanism of resistance for most tumours are likely to be multifactorial and the relationship between the microbiome, the immune system and the tumour microenvironment will play a role. $\mathrm{w}$

\section{Discussion and Conclusion}

Research regarding the role of gut microbiota in the treatment of advanced malignancy with ICls growing rapidly and presents possible mechanism for overcoming tumour resistance to $\mathrm{ICl}$ therapy, making a significant contribution to the ever-changing face of solid tumour treatments. Several factors have been identified that contribute towards positive responses, such

\section{References}

1 Migden MR (2018) PD-1 blockade with cemiplimab in advanced cutaneous squamous-cell carcinoma. N Engl J Med 379: 341-351.

2 Larkin J (2015) Combined nivolumab and ipilimumab or monotherapy in untreated melanoma. N Engl J Med 373: 23-34.

3 Robert C (2015) Pembrolizumab versus ipilimumab in advanced melanoma. N Engl J Med 372: 2521-2532.

4 Motzer RJ (2018) Nivolumab plus Ipilimumab versus Sunitinib in advanced renal-cell carcinoma. N Engl J Med 378: 1277-1290.

5 Seiwert TY (2016) Safety and clinical activity of pembrolizumab for treatment of recurrent or metastatic squamous cell carcinoma of the head and neck (KEYNOTE-012): an open-label, multicentre, phase 1b trial. Lancet Oncol 17: 956-965.

6 Zuur CL (2018) Immunomodulation by the combination of ipilimumab and nivolumab neoadjuvant to (salvage) surgery in advanced or recurrent head and neck carcinoma, IMCISION, an investigatorinitiated phase-Ib/II trial (N16IMC, NCT03003637). J Clin Oncol 36: e18020.

7 Antonia SJ (2016) Nivolumab alone and nivolumab plus ipilimumab in recurrent small-cell lung cancer (CheckMate 032): A multicentre, open-label, phase 1/2 trial. Lancet Oncol 17: 883-895.

8 Garon EB (2015) Pembrolizumab for the treatment of non-small-cell lung cancer. N Engl J Med 372: 2018-2028.

9 Huttenhower C (2012) Structure, function and diversity of the healthy human microbiome. Nature 486: 207.

10 Lepage PA (2013) metagenomic insight into our gut's microbiome. Gut 62: 146-158.

11 Markowiak P, Śliżewska KJGP (2018) The role of probiotics, prebiotics and synbiotic in animal nutrition. Gut Pathog 10: 21.

12 West NP (2013) Butyrylated starch increases colonic butyrate concentration but has limited effects on immunity in healthy physically active individuals. Exerc Immunol Rev 19: 102-119.

13 Azad M (2018) Probiotic species in the modulation of gut microbiota: an overview. Biomed Res Int 2018: 9478630.

14 Ford A (2014) Efficacy of prebiotics, probiotics, and synbiotics in irritable bowel syndrome and chronic idiopathic constipation: Systematic review and meta-analysis. Am J Gastroenterol 109: 1547. as increased biodiversity and specific bacteria populations in microbiomes. Opposing factors have also been acknowledged such as administration of antibiotics. This lends further support to the role of the faecal microbiome in host immunomodulation. Studies have also looked at various ways of administering interventions to manipulate the microbiome, including faecal transplants and short chain fatty acids via starches.

The next phase of research involves early interventional studies in a clinical context and a strong focus on biomarker development in these studies may also delineate the underlying mechanisms of immunomodulation by the microbiome. A number of clinical trials are underway utilising faecal transplantation in patients treated with immune checkpoint inhibitors for advanced malignancy. This represents a very exciting narrative in the unfolding journey of the treatment of many advanced malignancies.

15 Zhang H, Sun L (2018) When human cells meet bacteria: precision medicine for cancers using the microbiota. Am J Cancer Res 8: 1157.

16 Liu K, Chunwan L (2018) Gut microbes modulate host response to immune checkpoint inhibitor cancer immunotherapy. Transl Cancer Res 7: S608.

17 Trompette A (2014) Gut microbiota metabolism of dietary fiber influences allergic airway disease and hematopoiesis. Nat Med 20: 159.

18 Clarke JM (2007) Excretion of starch and esterified short-chain fatty acids by ileostomy subjects after the ingestion of acylated starches. Am J Clin Nutr 86: 1146-1151.

19 Leu RK (2015) Butyrylated starch intake can prevent red meatinduced 0 6-methyl-2-deoxyguanosine adducts in human rectal tissue: A randomised clinical trial. Br J Nutr 114: 220-230.

20 Humphreys KJ (2014) Dietary manipulation of oncogenic microRNA expression in human rectal mucosa: A randomized trial. Cancer Prev Res 7: 786-795.

21 Gill P (2018) Short chain fatty acids as potential therapeutic agents in human gastrointestinal and inflammatory disorders. Aliment Pharmacol Ther 48: 15-34.

22 Furusawa Y (2013) Commensal microbe-derived butyrate induces the differentiation of colonic regulatory T cells. Nature 504: 446.

23 Boussiotis V (2016) Molecular and biochemical aspects of the PD-1 checkpoint pathway. N Engl J Med 375: 1767-1778.

24 Routy B (2018) Gut microbiome influences efficacy of PD-1-based immunotherapy against epithelial tumors. Science 359: 91-97.

25 Sivan A (2015) Commensal Bifidobacterium promotes antitumor immunity and facilitates anti-PD-L1 efficacy. Science 350: 1084-1089.

26 Matson $V(2018)$ The commensal microbiome is associated with anti-PD-1 efficacy in metastatic melanoma patients. Science 359 : 104-108.

27 Vétizou M (2015) Anticancer immunotherapy by CTLA-4 blockade relies on the gut microbiota. Science 350: 1079-1084.

28 Gorjifard S, Goldszmid R (2016) Microbiota-myeloid cell crosstalk beyond the gut. J Leukoc Biol 100: 865-879.

29 Gopalakrishnan V (2018) Gut microbiome modulates response to anti-PD-1 immunotherapy in melanoma patients. Science 359: 97-103.

30 Langdon A, Crook N, Dantas G (2016) The effects of antibiotics on the 
microbiome throughout development and alternative approaches for therapeutic modulation. Genome Med 8: 39.

31 Derosa L (2018) Negative association of antibiotics on clinical activity of immune checkpoint inhibitors in patients with advanced renal cell and non-small-cell lung cancer. Ann Oncol 29: 1437-1444.

32 Brahmer JR (2018) Management of immune-related adverse events in patients treated with immune checkpoint inhibitor therapy: American Society of Clinical Oncology Clinical Practice Guideline. Clin Oncol 36: 1714-1768.

33 Ishikawa D (2016) Changes in intestinal microbiota following combination therapy with fecal microbial transplantation and antibiotics for ulcerative colitis. Inflamm Bowel Dis 23: 116-125.

34 Sokol H (2009) Low counts of Faecalibacterium prausnitzii in colitis microbiota. Inflamm Bowel Dis 15: 1183-1189.
35 Rabizadeh S (2007) Enterotoxigenic Bacteroides fragilis: A potential instigator of colitis. Inflamm Bowel Dis 13: 1475-1483.

36 Dubin K (2016) Intestinal microbiome analyses identify melanoma patients at risk for checkpoint-blockade-induced colitis. Nature Commun 7: 10391.

37 Chaput N (2017) Baseline gut microbiota predicts clinical response and colitis in metastatic melanoma patients treated with ipilimumab. Ann Oncol 28: 1368-1379.

38 Wang $Y$ (2018) Fecal microbiota transplantation for refractory immune checkpoint inhibitor-associated colitis. Nat Med 24: 18041808.

39 Rogado J (2019) Immune-related adverse events predict the therapeutic efficacy of anti-PD-1 antibodies in cancer patients. Eur J Cancer 109: 21-27. 